Preprint typeset in JHEP style - HYPER VERSION

MSUHEP-031216 hep-ph/0312324

\title{
Higgsless Electroweak Symmetry Breaking from Theory Space
}

\author{
Roshan Foadi, Shrihari Gopalakrishna, Carl Schmidt \\ Department of Physics and Astronomy, Michigan State University \\ East Lansing, MI 48824, USA \\ E-mail: foadi@pa.msu.edu, shri@pa.msu.edu, schmidt@pa.msu.edu
}

\begin{abstract}
We investigate unitarity of $W^{+} W^{-}$scattering in the context of theory space models of the form $U(1) \times[S U(2)]^{N} \times S U(2)_{N+1}$, which are broken down to $U(1)_{E M}$ by non-linear $\Sigma$ fields, without the presence of a physical Higgs Boson. By allowing the couplings of the $U(1)$ and the final $S U(2)_{N+1}$ to vary, we can fit the $W$ and $Z$ masses, and we find that the coefficient of the term in the amplitude that grows as $E^{2} / m_{W}^{2}$ at high energies is suppressed by a factor of $(N+1)^{-2}$. In the $N+1 \rightarrow \infty$ limit the model becomes a 5 -dimensional $S U(2)$ gauge theory defined on an interval, where boundary terms at the two ends of the interval break the $S U(2)$ down to $U(1)_{E M}$. These boundary terms also modify the Kaluza-Klein (KK) mass spectrum, so that the lightest KK states can be identified as the $W$ and $Z$ bosons. The $T$ parameter, which measures custodial symmetry breaking, is naturally small in these models. Depending on how matter fields are included, the strongest experimental constraints come from precision electroweak limits on the $S$ parameter.
\end{abstract}

Keywords: Beyond Standard Model, Spontaneous Symmetry Breaking. 


\section{Contents}

1. Introduction 1

2. $U(1) \times S U(2)_{1} \times S U(2)_{2}$ Model 3

3. $U(1) \times[S U(2)]^{N} \times S U(2)_{N+1}$ Model

4. The $N+1 \rightarrow \infty$ limit 10

5. Coupling to matter fields and constraints from experiment 12

6. Conclusions 15

A. Solutions for the $U(1) \times S U(2)_{1} \times S U(2)_{2}$ Model 16

B. Solutions for the $U(1) \times[S U(2)]^{N} \times S U(2)_{N+1}$ Model

\section{Introduction}

Despite the successes of the standard model of particle physics, we know that it is incomplete without some, as yet, undiscovered particles and interactions. The simplest argument for this is to consider the scattering of longitudinal vector bosons at high energies. The scattering amplitude for $W_{L}^{+} W_{L}^{-} \rightarrow W_{L}^{+} W_{L}^{-}$grows as $E^{2} / m_{W}^{2}$ at high energies, and therefore violates unitarity at some scale. This is the main motivation for the existence of one or more Higgs scalars, which naturally fix this problem by providing new contributions to the scattering amplitude [1]. The perturbative breaking of the $S U(2) \times U(1)$ symmetry by Higgs vacuum expectation values (vevs) is intimately related with this unitarity restoration. An alternative path to symmetry breaking is some new strong dynamics, such as technicolor [2]. In these types of models the unitarity is restored through the exchange of composite states, such as techni-rho mesons.

Recently, a new mechanism for gauge-symmetry breaking has been suggested [3, 47. This involves the embedding of the 4-dimensional theory in some higher-dimensional model, where the gauge symmetry is exact in the bulk of the extra dimensions, but it is explicitly broken on the boundaries. The breaking can either be due to orbifolding the extra dimensions or to simply imposing conditions on the fields at the boundary, which distinguish between subsets of the full gauge group [4]. Despite the explicit symmetry breaking in the higher-dimensional theory, it appears soft in the effective 4-dimensional theory, in 
the sense that the unitarity of scattering amplitudes is maintained [5].* In this case the unitarity of the longitudinal vector boson scattering amplitude is restored via the exchange of Kaluza-Klein (KK) excitations of the gauge bosons.

Models of this sort have been used to explain the breaking of a grand unified gauge group down to the standard model $S U(3) \times S U(2) \times U(1)$. (See [3], for example.) Attempts have also been made to use this mechanism to break the electroweak gauge group $S U(2) \times$ $U(1)$ down to electromagnetism $U(1)_{E M}[6,7,7,8]$ without the necessity for a Higgs scalar. In this paper we use the technique of deconstruction of the extra dimension [9] as an aid to addressing this second idea. In deconstruction of a five-dimensional gauge theory the fifth dimension is discretized, and the gauge fields $A^{\mu}$ at each position in the extra dimension become independent gauge fields of a product gauge group in four dimensions. The gauge fields that point along the fifth dimension, $A^{5}$, are reinterpreted as the Goldstone-boson fields of a non-linear sigma model, which break the gauge groups at neighboring sites of the discretized extra dimension down to the diagonal. We shall see that investigating deconstructed models offers a new insight into this mechanism of symmetry breaking and leads us to a new model, which is arguably simpler than those proposed previously, even after the continuum limit is recovered.

A useful example of deconstruction of a theory with symmetry-breaking boundary conditions is the $S U(2)$ model of Ref. [4], defined on a fifth-dimensional interval, $0 \leq y \leq$ $\pi R$, where the $S U(2)$ group is broken down to $U(1)$ at $y=0$ by boundary conditions. The deconstruction of this model is a $U(1) \times[S U(2)]^{N+1}$ gauge theory with sigma model fields that break the $S U(2) \times S U(2)$ at neighboring sites down to the diagonal. (The $U(1)$ at the first site is realized by gauging the $U(1)$ subgroup of a global $S U(2)$.) An interesting observation is that the $N=0$ case of this deconstructed theory just corresponds to the electroweak gauge group, except that in the deconstructed model the $U(1)$ and $S U(2)$ couplings are identical. If we allow the couplings to be different, we have the effective field theory for a standard model in which the electroweak symmetry is broken strongly, such as technicolor. Thus, for general $N$, one might consider whether the good unitarity properties of the deconstructed theory can be maintained, while relaxing the conditions on the gauge couplings of the product groups and on the vevs of the sigma model fields. In the remainder of this paper, we follow this line of reasoning and see where it leads.

In section 2 we consider the simplest extension of the (Higgsless) standard model of this type, which is $U(1) \times S U(2)_{1} \times S U(2)_{2}$. Allowing the three couplings and the two vacuum expectation values (which break the group down to $U(1)_{E M}$ ) to be independent, we can fit the lightest charged and neutral vector bosons to the $W$ and $Z$ masses and the remnant gauge coupling to the electromagnetic coupling $e$. We then investigate the question of unitarity of the $W_{L}^{+} W_{L}^{-} \rightarrow W_{L}^{+} W_{L}^{-}$scattering amplitude as a function of the two remaining parameters, which can be taken to be the masses of the $W^{\prime}$ and $Z^{\prime}$ which arise in this theory. We find that the unitarity violation is postponed to the greatest extent when the two vevs of the sigma fields are roughly equal. When this condition is satisfied,

\footnotetext{
* Of course, at sufficiently high energies, unitarity problems will reappear, due to the fact that the higherdimensional theory is not renormalizable. Although this must be addressed in a complete self-consistent theory, it is beyond the scope of the ideas considered in this paper.
} 


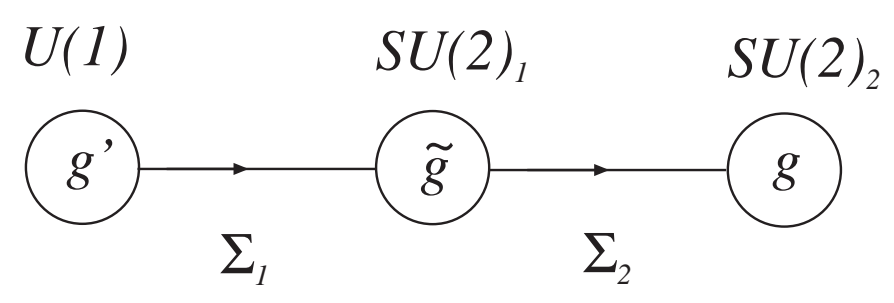

Figure 1: Moose diagram for $U(1) \times S U(2)_{1} \times S U(2)_{2}$ model.

we find that $m_{W^{\prime}}$ and $m_{Z^{\prime}}$ are approximately proportional to the coupling of the central $S U(2)_{1}$, while the $W$ and $Z$ masses and couplings are essentially given by those of the $U(1)$ and $S U(2)_{2}$ on the ends of the chain.

Motivated by those results, we then consider in section 3 the generalization to $U(1) \times$ $[S U(2)]^{N} \times S U(2)_{N+1}$, where all of the vevs of the sigma fields are taken identical, and all of the couplings except for those of the $U(1)$ and the $S U(2)_{N+1}$ on the end are taken identical. We find that in the limit of $N+1 \rightarrow \infty$ the same good unitarity behavior of the extra-dimensional theory is recovered. In section 4 we consider the $N+1 \rightarrow \infty$ limit further and examine the five-dimensional theory that is obtained. In section 5 we investigate the question of the inclusion of fermions in these models, and analyze the direct and indirect constraints from experiment. Finally, in section 6 we give our conclusions. We also have included two appendices which give more details of the solutions to the $N=1$ and general $N$ models considered in sections 2 and 3 , respectively.

\section{2. $U(1) \times S U(2)_{1} \times S U(2)_{2}$ Model}

We begin with the theory space model with the group structure $U(1) \times S U(2)_{1} \times S U(2)_{2}$ and defined by the moose diagram [10] of Fig. 1, where the $U(1)$ is treated as the $U(1)$ subgroup of a global $S U(2)$ group. The nonlinear sigma model fields,

$$
\Sigma_{1}=e^{2 i \pi_{1}^{a} T^{a} / f_{1}}, \quad \Sigma_{2}=e^{2 i \pi_{2}^{a} T^{a} / f_{2}}
$$

consist of two $S U(2)$ triplets, which are coupled to the gauge fields by the covariant derivatives

$$
\begin{aligned}
& D_{\mu} \Sigma_{1}=\partial_{\mu} \Sigma_{1}-i g^{\prime} T^{3} B_{\mu} \Sigma_{1}+i \tilde{g} \Sigma_{1} T^{a} W_{1 \mu}^{a}, \\
& D_{\mu} \Sigma_{2}=\partial_{\mu} \Sigma_{2}-i \tilde{g} T^{a} W_{1 \mu}^{a} \Sigma_{2}+i g \Sigma_{2} T^{a} W_{2 \mu}^{a} .
\end{aligned}
$$

The Lagrangian density for the relevant fields is

$$
\begin{aligned}
\mathcal{L}= & -\frac{1}{4} B^{\mu \nu} B_{\mu \nu}-\frac{1}{4} W_{1}^{a \mu \nu} W_{1 \mu \nu}^{a}-\frac{1}{4} W_{2}^{a \mu \nu} W_{2 \mu \nu}^{a} \\
& +\frac{f_{1}^{2}}{4} \operatorname{tr}\left[D^{\mu} \Sigma_{1}\left(D_{\mu} \Sigma_{1}\right)^{\dagger}\right]+\frac{f_{2}^{2}}{4} \operatorname{tr}\left[D^{\mu} \Sigma_{2}\left(D_{\mu} \Sigma_{2}\right)^{\dagger}\right] .
\end{aligned}
$$

After the sigma fields acquire a vacuum expectation value, $\left\langle\Sigma_{i}\right\rangle=1$, their only affect in unitary gauge is to give mass to the $W$ 's and $Z$ 's. 
In this model there are five independent parameters: $g^{\prime}, \tilde{g}, g, f_{1}, f_{2}$. Since we want to recover the standard model at low energy, we can exchange three of these for the electromagnetic coupling $e$, and the $W$ and $Z$ boson masses, $m_{W}$ and $m_{Z}$. (Note that we are only working at tree level throughout this paper.) It is convenient to take the masses of the heavy $W^{\prime}$ and $Z^{\prime}$ bosons, $m_{W^{\prime}}$ and $m_{Z^{\prime}}$, as the remaining independent parameters. The charged gauge bosons can be expanded in terms of the mass eigenstates by

$$
\begin{aligned}
& W_{1}^{ \pm}=a_{11} W^{\prime \pm}+a_{12} W^{ \pm} \\
& W_{2}^{ \pm}=a_{21} W^{\prime \pm}+a_{22} W^{ \pm} .
\end{aligned}
$$

The neutral bosons can be expanded in terms of mass eigenstates by

$$
\begin{aligned}
B & =b_{00} A+b_{01} Z^{\prime}+b_{02} Z \\
W_{1}^{3} & =b_{10} A+b_{11} Z^{\prime}+b_{12} Z \\
W_{2}^{3} & =b_{20} A+b_{21} Z^{\prime}+b_{22} Z,
\end{aligned}
$$

where the photon $A$ is massless. Formulae for the original parameters $g^{\prime}, \tilde{g}, g, f_{1}, f_{2}$, and the mixing matrices, $a_{i j}, b_{i j}$ as functions of the independent variables $e, m_{W}, m_{Z}, m_{W^{\prime}}, m_{Z^{\prime}}$ can be found in Appendix A.

We now would like to see if it is possible to cancel the bad high energy behavior in the $W_{L}^{+} W_{L}^{-}$scattering amplitude, or at least reduce it, for some choice of parameters in our model. In particular we are concerned with the growth proportional to $E^{2} / m_{W}^{2}$ for high energies in the $W_{L}^{+} W_{L}^{-} \rightarrow W_{L}^{+} W_{L}^{-}$amplitude. The amplitude can be written

$$
\begin{aligned}
\mathcal{A}=\frac{1}{m_{W}^{4}} & \left\{\left(\tilde{g}^{2} a_{12}^{4}+g^{2} a_{22}^{4}\right)\left[p^{2} E^{2}(-2+6 \cos \theta)+E^{4}\left(-1+\cos ^{2} \theta\right)\right]\right. \\
+ & {\left[-p^{2} \cos \theta\left(p^{2}-3 E^{2}\right)^{2}\right] } \\
\quad & {\left[\frac{e^{2}}{s}+\frac{\left(\tilde{g} b_{12} a_{12}^{2}+g b_{22} a_{22}^{2}\right)^{2}}{s-m_{Z}^{2}}+\frac{\left(\tilde{g} b_{11} a_{12}^{2}+g b_{21} a_{22}^{2}\right)^{2}}{s-m_{Z^{\prime}}^{2}}\right] } \\
- & {\left[4 E^{2}\left(p^{2}+\left(E^{2}-2 p^{2}\right) \cos \theta\right)^{2}+2 p^{2}(1+\cos \theta)\left(2 E^{2}-p^{2}-E^{2} \cos \theta\right)^{2}\right] } \\
& \left.\times\left[\frac{e^{2}}{t}+\frac{\left(\tilde{g} b_{12} a_{12}^{2}+g b_{22} a_{22}^{2}\right)^{2}}{t-m_{Z}^{2}}+\frac{\left(\tilde{g} b_{11} a_{12}^{2}+g b_{21} a_{22}^{2}\right)^{2}}{t-m_{Z^{\prime}}^{2}}\right]\right\}
\end{aligned}
$$

where the energy $E$, momentum $p=\left(E^{2}-m_{W}^{2}\right)^{1 / 2}$, and scattering angle $\theta$ of the $W$ 's are in the center of momentum frame, while $s=4 E^{2}$ and $t=-2 p^{2}(1-\cos \theta)$ are the standard Mandelstam variables.

At high energies the amplitude can be expanded in powers of $m_{W}^{2} / E^{2}$. The coefficient of the leading $E^{4} / m_{W}^{4}$ vanishes due to gauge invariance. We are then left with

$$
\mathcal{A}=\frac{E^{2}}{m_{W}^{2}} \frac{(1+\cos \theta)}{2} R+\mathcal{O}\left(\left(m_{W}^{2} / E^{2}\right)^{0}\right),
$$




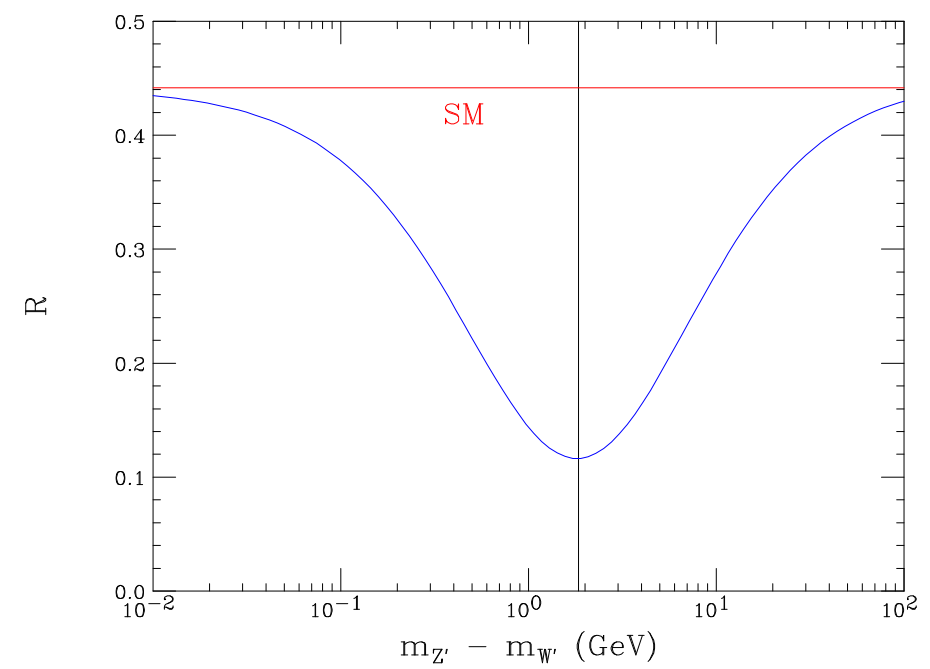

Figure 2: The coefficient of the leading $E^{2} / m_{W}^{2}$ term in the $W^{+} W^{-} \rightarrow W^{+} W^{-}$scattering amplitude in the $U(1) \times S U(2)_{1} \times S U(2)_{2}$ model (blue) as a function of the $Z^{\prime}$ and $W^{\prime}$ mass difference, with $m_{W^{\prime}}=500 \mathrm{GeV}$ fixed. The same quantity in the standard model without a Higgs boson (red) is also plotted. The vertical line indicates the position where $m_{Z^{\prime}}^{2}-m_{W^{\prime}}^{2}=m_{Z}^{2}-m_{W}^{2}$.

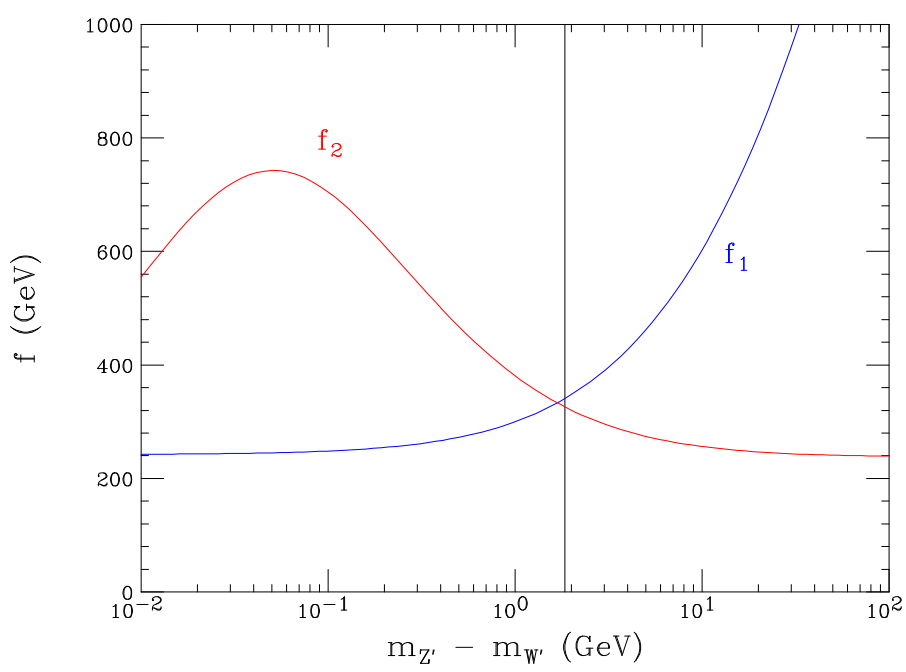

Figure 3: The quantities $f_{1}$ (blue) and $f_{2}$ (red) as a function of the $Z^{\prime}$ and $W^{\prime}$ mass difference, with $m_{W^{\prime}}=500 \mathrm{GeV}$ fixed. The vertical line indicates the position where $m_{Z^{\prime}}^{2}-m_{W^{\prime}}^{2}=m_{Z}^{2}-m_{W}^{2}$.

where

$$
R=4\left(\tilde{g}^{2} a_{12}^{4}+g^{2} a_{22}^{4}\right)-3\left[\left(\tilde{g} b_{12} a_{12}^{2}+g b_{22} a_{22}^{2}\right)^{2} \frac{m_{Z}^{2}}{m_{W}^{2}}+\left(\tilde{g} b_{11} a_{12}^{2}+g b_{21} a_{22}^{2}\right)^{2} \frac{m_{Z^{\prime}}^{2}}{m_{W}^{2}}\right] .
$$

Using the formulae in Appendix A, we can treat $R \equiv R\left(m_{W^{\prime}}, m_{Z^{\prime}}\right)$ as a function of $m_{W^{\prime}}$ and $m_{Z^{\prime}}$.

In Fig. 2 we plot $R$ as a function of the mass difference, $m_{Z^{\prime}}-m_{W^{\prime}}$, for $m_{W^{\prime}}=500$ $\mathrm{GeV}$ fixed. As a comparison we also plot the same quantity in the standard model without 


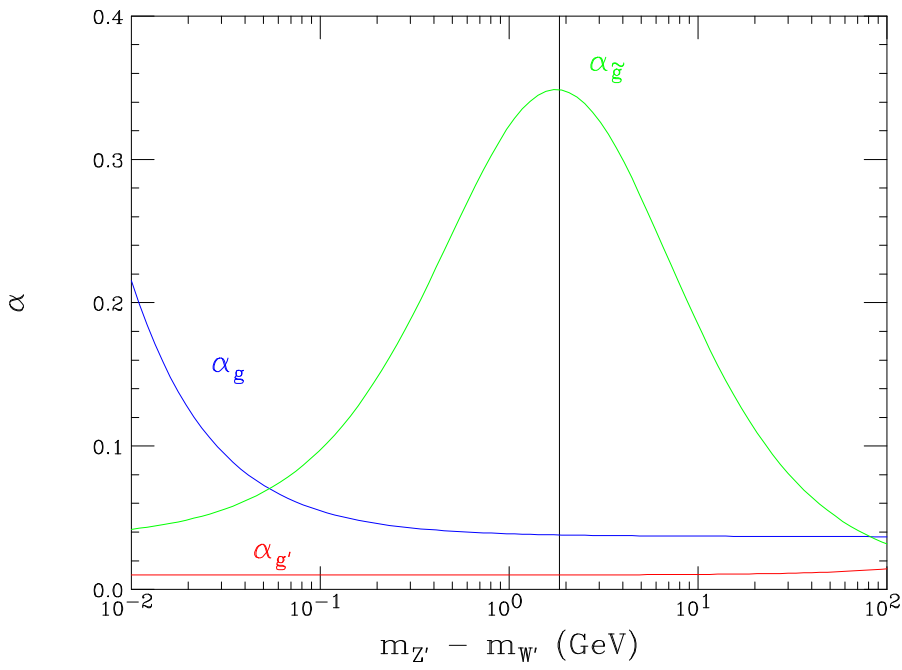

Figure 4: The coupling constants $\alpha_{g}=g^{2} / 4 \pi$ (blue), $\alpha_{g^{\prime}}=g^{\prime 2} / 4 \pi$ (red), and $\alpha_{\tilde{g}}=\tilde{g}^{2} / 4 \pi$ (green), as a function of the $Z^{\prime}$ and $W^{\prime}$ mass difference, with $m_{W^{\prime}}=500 \mathrm{GeV}$ fixed. The vertical line indicates the position where $m_{Z^{\prime}}^{2}-m_{W^{\prime}}^{2}=m_{Z}^{2}-m_{W}^{2}$.

the Higgs boson. Note that $R$ is significantly suppressed for $m_{Z^{\prime}}^{2}-m_{W^{\prime}}^{2} \approx m_{Z}^{2}-m_{W}^{2}$. When this relation holds, the value of $R$ is reduced by almost precisely a factor of $1 / 4$, a result which does not depend on the particular value of $m_{W^{\prime}}$. This indicates that the unitarity violation that occurs in the standard model without a Higgs boson would be postponed to higher energy in this model. We also plot in Fig. 3 the scales $f_{1}$ and $f_{2}$, and in Fig. 4 the couplings constants $\alpha_{g}=g^{2} / 4 \pi, \alpha_{g^{\prime}}=g^{\prime 2} / 4 \pi$, and $\alpha_{\tilde{g}}=\tilde{g}^{2} / 4 \pi$, as a function of the $Z^{\prime}$ and $W^{\prime}$ mass difference, with $m_{W^{\prime}}=500 \mathrm{GeV}$ fixed. We note that the relation $m_{Z^{\prime}}^{2}-m_{W^{\prime}}^{2} \approx m_{Z}^{2}-m_{W}^{2}$ also corresponds to $f_{1} \approx f_{2}$ and $\tilde{g} \gg g, g^{\prime}$. In fact when this relation holds the couplings are given to a good approximation by $g=e / \sin \theta_{W}$, $g^{\prime}=e / \cos \theta_{W}$, and $\tilde{g}=\left(m_{W^{\prime}} / 2 m_{W}\right) g$, up to corrections of order $m_{W}^{2} / m_{W^{\prime}}^{2}$. (We have used the tree level definition of $\cos \theta_{W}=m_{W} / m_{Z}$.) Thus, the $S U(2)_{2}$ and the $U(1)$ act approximately like the $S U(2)_{L}$ and $U(1)_{Y}$ of the standard model, while the intervening $S U(2)_{1}$ has the effect of softening the unitarity violation of the standard model $W_{L} W_{L}$ scattering.

We can observe the effect of the delayed unitary violation by plotting the $J=0$ partial wave amplitude,

$$
a_{0}=\frac{1}{32 \pi} \int_{-1}^{1} \mathcal{A} d \cos \theta
$$

as a function of $\sqrt{s}=2 E$. This is shown in Fig. 0 for both the standard model without a Higgs boson and in the $U(1) \times S U(2)_{1} \times S U(2)_{2}$ model with $m_{W^{\prime}}=500 \mathrm{GeV}$ and $m_{Z^{\prime}}^{2}-m_{W^{\prime}}^{2}=m_{Z}^{2}-m_{W}^{2}$. (We have included a photon mass of $m_{A}=1 \mathrm{GeV}$ in order to regulate the $t$-channel singularity in the integral of Eq. (2.9). This is inconsequential in the high energy region in which we are interested.) Since unitarity requires $\left|\operatorname{Re} a_{0}\right|<1 / 2$, we can use this figure to infer that unitarity violation in this amplitude has been postponed from a scale of $\sqrt{s} \approx 1.6 \mathrm{TeV}$ in the standard model without a Higgs boson to $\sqrt{s} \approx 2.65$ 


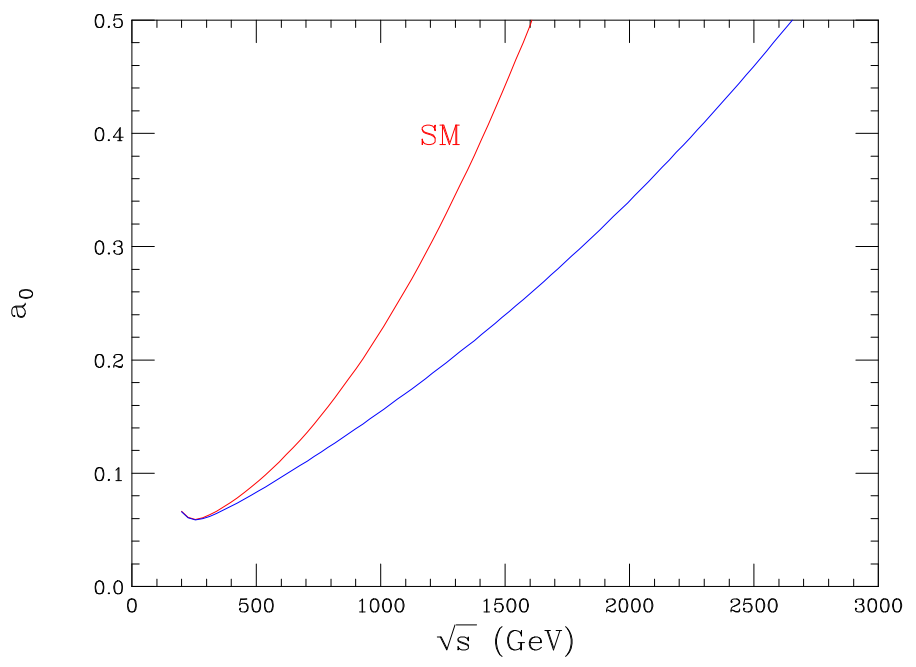

Figure 5: The $J=0$ partial wave amplitude as a function of $\sqrt{s}$ for the standard model without a Higgs boson (red) and the $U(1) \times S U(2)_{1} \times S U(2)_{2}$ model (blue) with $m_{W^{\prime}}=500 \mathrm{GeV}$ and $m_{Z^{\prime}}^{2}-m_{W^{\prime}}^{2}=m_{Z}^{2}-m_{W}^{2}$.

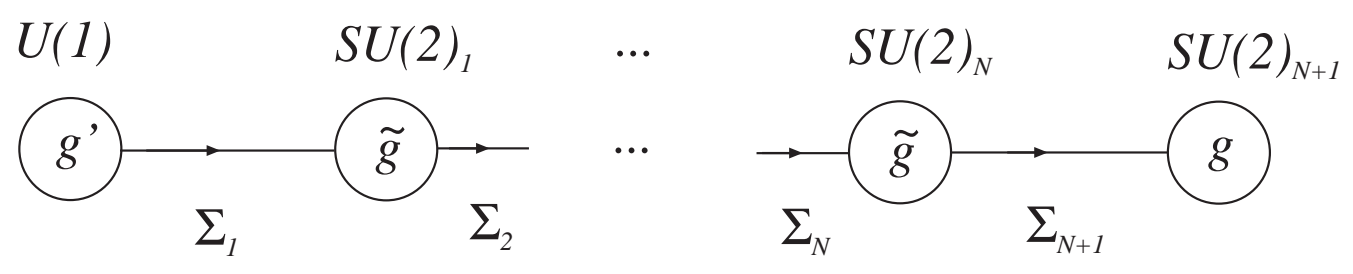

Figure 6: Moose diagram for $U(1) \times[S U(2)]^{N} \times S U(2)_{N+1}$ model.

$\mathrm{TeV}$ in the $U(1) \times S U(2)_{1} \times S U(2)_{2}$ model with this choice of parameters.

We have found that the behavior of the $W_{L}^{+} W_{L}^{-} \rightarrow Z_{L} Z_{L}$ amplitude to be essentially identical to that for $W_{L}^{+} W_{L}^{-} \rightarrow W_{L}^{+} W_{L}^{-}$. In particular the corresponding value of $R$, the coefficient of the leading $E^{2} / m_{W}^{2}$ term in that amplitude, is reduced by the same factor of $1 / 4$ when $m_{Z^{\prime}}^{2}-m_{W^{\prime}}^{2} \approx m_{Z}^{2}-m_{W}^{2}$.

\section{3. $U(1) \times[S U(2)]^{N} \times S U(2)_{N+1}$ Model}

In section 2 we saw that the choice of parameters which produced the greatest postponement of unitarity led to a model where the standard model $S U(2)_{L}$ and $U(1)_{Y}$ gauge groups were separated in theory space by an extra intervening $S U(2)$. Following the extradimensional analogue further, we now extend this to a series of intervening $S U(2)$ 's, all with the same coupling and all vevs chosen to be the same. The moose diagram for this theory is shown in Fig. 6 .

The Lagrange density for this model is

$$
\mathcal{L}=-\frac{1}{4} B^{\mu \nu} B_{\mu \nu}-\frac{1}{4} \sum_{i=1}^{N+1} W_{i}^{a \mu \nu} W_{i \mu \nu}^{a}+\frac{f^{2}}{4} \sum_{i=1}^{N+1} \operatorname{tr}\left[D^{\mu} \Sigma_{i}\left(D_{\mu} \Sigma_{i}\right)^{\dagger}\right],
$$


and the nonlinear sigma model fields can be parameterized by

$$
\Sigma_{i}=e^{2 i \pi_{i}^{a} T^{a} / f} .
$$

The $\Sigma_{i}$ 's are coupled to the gauge fields by the covariant derivatives

$$
\begin{aligned}
D_{\mu} \Sigma_{1} & =\partial_{\mu} \Sigma_{1}-i g^{\prime} T^{3} B_{\mu} \Sigma_{1}+i \tilde{g} \Sigma_{1} T^{a} W_{1 \mu}^{a}, \\
D_{\mu} \Sigma_{k} & =\partial_{\mu} \Sigma_{k}-i \tilde{g} T^{a} W_{k-1 \mu}^{a} \Sigma_{k}+i \tilde{g} \Sigma_{k} T^{a} W_{k \mu}^{a}, \quad(k \neq 1, N+1) \\
D_{\mu} \Sigma_{N+1} & =\partial_{\mu} \Sigma_{N+1}-i \tilde{g} T^{a} W_{N \mu}^{a} \Sigma_{N+1}+i g \Sigma_{N+1} T^{a} W_{N+1 \mu}^{a} .
\end{aligned}
$$

As in the previous model, the sigma fields can be removed in unitary gauge, giving a mass to the $W$ 's and $Z$ 's. We can then expand the charged fields in terms of the mass eigenstates

$$
W_{j}^{ \pm}=\sum_{k=1}^{N} a_{j k} W_{k}^{ \pm}+a_{j(N+1)} W^{ \pm},
$$

and similarly for the neutral fields

$$
\begin{gathered}
B=b_{00} A+\sum_{k=1}^{N} b_{0 k} Z_{k}^{\prime}+b_{0(N+1)} Z \\
W_{j}^{3}=b_{j 0} A+\sum_{k=1}^{N} b_{j k} Z_{k}^{\prime}+b_{j(N+1)} Z,
\end{gathered}
$$

where the photon $A$ is exactly massless as required.

We give the general solution for the diagonalization of these mass matrices in Appendix $\mathrm{B}$. For this model there are four independent parameters, $g^{\prime}, \tilde{g}, g$, $f$, which can be fixed by $e, m_{W}, m_{Z}$, and the mass of the lightest $W^{\prime}, m_{W_{1}^{\prime}}$. If we assume that $\tilde{g} \gg g, g^{\prime}$, and letting $\lambda^{2}=g^{2} / \tilde{g}^{2}$ and $\lambda^{\prime 2}=g^{\prime 2} / \tilde{g}^{2}$, we obtain the masses

$$
\begin{aligned}
m_{W}^{2} & =\frac{g^{2} f^{2}}{4(N+1)}\left(1+\mathcal{O}\left(\lambda^{2}\right)\right) \\
m_{Z}^{2} & =\frac{\left(g^{2}+g^{\prime 2}\right) f^{2}}{4(N+1)}\left(1+\mathcal{O}\left(\lambda^{2}\right)\right) \\
m_{W_{k}^{\prime}}^{2} & =\tilde{g}^{2} f^{2}\left(\sin \frac{k \pi}{2(N+1)}\right)^{2}+2 m_{W}^{2}\left(\cos \frac{k \pi}{2(N+1)}\right)^{2}\left(1+\mathcal{O}\left(\lambda^{2}\right)\right) \\
m_{Z_{k}^{\prime}}^{2} & =\tilde{g}^{2} f^{2}\left(\sin \frac{k \pi}{2(N+1)}\right)^{2}+2 m_{Z}^{2}\left(\cos \frac{k \pi}{2(N+1)}\right)^{2}\left(1+\mathcal{O}\left(\lambda^{2}\right)\right) .
\end{aligned}
$$

It is easy to check that for $N=1$ this gives $m_{Z_{1}^{\prime}}^{2}-m_{W_{1}^{\prime}}^{2} \approx m_{Z}^{2}-m_{W}^{2}$, and $\tilde{g}=\left(m_{W_{1}^{\prime}} / 2 m_{W}\right) g$, up to corrections of order $m_{W}^{2} / m_{W_{1}^{\prime}}^{2}$, as found in the previous section.

The scattering of longitudinal $W$ 's is easily generalized from the last section. The amplitude for $W_{L}^{+} W_{L}^{-} \rightarrow W_{L}^{+} W_{L}^{-}$is given by

$$
\mathcal{A}=\frac{1}{m_{W}^{4}}\left\{\left(\sum_{i=1}^{N+1} g_{i}^{2} a_{i(N+1)}^{4}\right)\left[p^{2} E^{2}(-2+6 \cos \theta)+E^{4}\left(-1+\cos ^{2} \theta\right)\right]\right.
$$




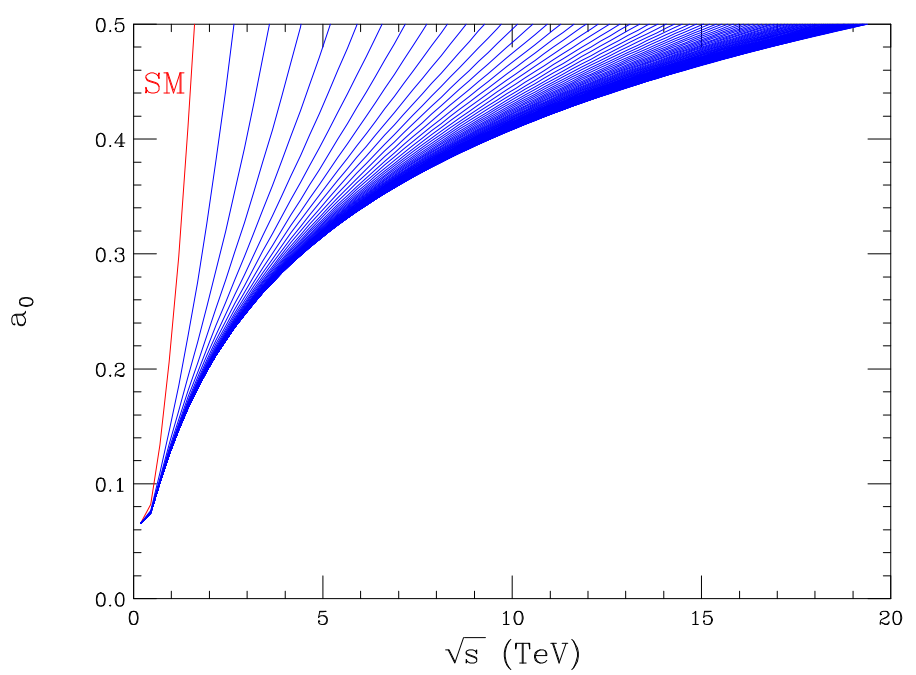

Figure 7: The $J=0$ partial wave amplitude as a function of $\sqrt{s}$ for the standard model without a Higgs boson (red) and the $U(1) \times[S U(2)]^{N} \times S U(2)_{N+1}$ model (blue) for $N=1$ to 100 with $m_{W_{1}^{\prime}}=500 \mathrm{GeV}$.

$$
\begin{aligned}
& +\left[-p^{2} \cos \theta\left(p^{2}-3 E^{2}\right)^{2}\right]\left[\frac{e^{2}}{s}+\sum_{k=1}^{N+1} \frac{\left(\sum_{i=1}^{N+1} g_{i} b_{i k} a_{i(N+1)}^{2}\right)^{2}}{s-m_{Z_{k}^{\prime}}^{2}}\right] \\
& -\left[4 E^{2}\left(p^{2}+\left(E^{2}-2 p^{2}\right) \cos \theta\right)^{2}+2 p^{2}(1+\cos \theta)\left(2 E^{2}-p^{2}-E^{2} \cos \theta\right)^{2}\right] \\
& \left.\times\left[\frac{e^{2}}{t}+\sum_{k=1}^{N+1} \frac{\left(\sum_{i=1}^{N+1} g_{i} b_{i k} a_{i(N+1)}^{2}\right)^{2}}{t-m_{Z_{k}^{\prime}}^{2}}\right]\right\} .
\end{aligned}
$$

In this formula we have defined $g_{i}=\tilde{g}$ for $i \neq N+1$ and $g_{N+1}=g$, and we have identified $Z_{N+1}^{\prime} \equiv Z$ in order to make it more compact. Then the coefficient of the leading $E^{2} / m_{W}^{2}$ term, defined by Eq. (2.7) is

$$
R=4\left(\sum_{i=1}^{N+1} g_{i}^{2} a_{i(N+1)}^{4}\right)-3\left[\sum_{k=1}^{N+1}\left(\sum_{i=1}^{N+1} g_{i} b_{i k} a_{i(N+1)}^{2}\right)^{2} \frac{m_{Z_{k}^{\prime}}^{2}}{m_{W}^{2}}\right] .
$$

In Appendix B we obtain for this model

$$
\begin{aligned}
R & =\frac{g^{2}}{(N+1)^{2}}+\mathcal{O}\left(\lambda^{2}\right) \\
& =\frac{R(S M)}{(N+1)^{2}}+\mathcal{O}\left(\lambda^{2}\right) .
\end{aligned}
$$

where the corrections also fall off as $(N+1)^{-2}$. As expected, this agrees with the results of the previous section for $N=1$.

In Fig. 7 we plot the $J=0$ partial wave amplitude as a function of $\sqrt{s}$ for both the standard model without a Higgs boson and in the $U(1) \times[S U(2)]^{N} \times S U(2)_{N+1}$ model with $m_{W_{1}^{\prime}}=500 \mathrm{GeV}$ for $N=1$ to 100 . For large $N$ in this model the unitarity violation 
is delayed to an energy of about $\sqrt{s}=19 \mathrm{TeV}$. Thus, we may expect that the effective theory with a KK tower of vector bosons should be reliable up to about this scale. ${ }^{\dagger}$ At high energies and large $N$, the partial wave amplitude asymptotes to

$$
\begin{aligned}
a_{0} & \approx \frac{1}{32 \pi}\left[\frac{s}{4 m_{W}^{2}} \frac{g^{2}}{(N+1)^{2}}+\frac{4 \tilde{g}^{2}}{N+1}\left(\ln \frac{s}{\Lambda^{2}}-\frac{1}{2}\right)\right] \\
& \approx \frac{1}{32 \pi}\left[\frac{s}{4 m_{W}^{2}} \frac{g^{2}}{(N+1)^{2}}+\frac{4 g^{2}}{\pi^{2}} \frac{m_{W_{1}^{\prime}}^{2}}{m_{W}^{2}}\left(\ln \frac{s}{\Lambda^{2}}-\frac{1}{2}\right)\right]
\end{aligned}
$$

where $\Lambda$ is a scale on the order of a few times $m_{W_{1}^{\prime}}$, the mass of the lightest $W^{\prime}$.

\section{The $N+1 \rightarrow \infty$ limit}

We now consider the $N+1 \rightarrow \infty$ limit of this model. For large $N$ the sites in the moose diagram of Fig. 6 play the role of lattice sites in a fifth dimension, and the model behaves as a latticized extra-dimensional theory [9]. Taking the lattice spacing to be $a$ and the length of the extra dimension to be $\pi R$, we can relate these to the parameters in the model by

$$
\pi R=(N+1) a=\frac{2(N+1)}{\tilde{g} f} .
$$

The five-dimensional gauge coupling in the bulk is related to the four-dimensional coupling by $g_{5}^{2}=a \tilde{g}^{2}$, which gives

$$
\frac{\tilde{g}^{2}}{N+1}=\frac{g_{5}^{2}}{\pi R}
$$

Thus, we find that both the coupling $\tilde{g}$ and the parameter $f$ scale as $(N+1)^{1 / 2}$ as $N+1 \rightarrow$ $\infty$. In this limit the model becomes a five-dimensional $S U(2)$ gauge theory, where the fifth dimension is the line segment, $0 \leq y \leq \pi R$. In addition there are boundary terms at $y=0$ and $y=\pi R$, with the boundary conditions at $y=0$ breaking the gauge symmetry down to $U(1)$. The five dimensional action is

$$
\begin{aligned}
\mathcal{S}=\int_{0}^{\pi R} d y \int d^{4} x[ & -\frac{1}{4 g_{5}^{2}} W^{a M N} W_{M N}^{a}-\delta(y-\pi R) \frac{1}{4 g^{2}} W^{a \mu \nu} W_{\mu \nu}^{a} \\
& \left.-\delta(y) \frac{1}{4 g^{\prime 2}} W^{3 \mu \nu} W_{\mu \nu}^{3}\right]
\end{aligned}
$$

where, in this equation, the indices $M, N$ run over the 5 dimensions, and we impose the Dirichlet Boundary condition, $W_{\mu}^{a}=0$, at $y=0$ for $a \neq 3$. The $\delta$-function at $y=\pi R$ should be interpreted as $\delta(y-\pi R+\epsilon)$ with $\epsilon \rightarrow 0^{+}$and the fields having Neumann boundary conditions, $d W_{\mu}^{a} / d y=0$, at $y=\pi R$. The $\delta$-function and the field $W_{\mu}^{3}$ at $y=0$ should be interpreted similarly.

\footnotetext{
${ }^{\dagger}$ A coupled-channel analysis, as considered in Ref. [⿰] [], would give a lower energy scale for unitarity violation.
} 
All of the results found in appendix $\mathrm{B}$ have a well-defined limit as $N+1 \rightarrow \infty$. The tower of massive vector particles becomes a tower of Kaluza-Klein states given by the decomposition

$$
W_{\mu}^{ \pm}(x, y)=\sum_{n=0}^{\infty} C_{(n)} \sin \left(m_{W_{n}^{\prime}} y\right) W_{n, \mu}^{ \pm}(x),
$$

where $C_{(n)}$ is a normalization factor, and the masses $m_{W_{n}^{\prime}}$ for the $W^{\prime \pm}$ states are the solutions of

$$
m_{W_{n}^{\prime}} \tan \left(m_{W_{n}^{\prime}} \pi R\right)=\frac{g^{2}}{g_{5}^{2}}
$$

The lightest of these charged vector mesons is just the standard model $W^{ \pm} \equiv W_{0}^{\prime \pm}$, with mass $m_{W} \approx g /\left(g_{5} \sqrt{\pi R}\right)$.

Similarly the neutral vector bosons can be expanded in Kaluza-Klein states

$$
W_{\mu}^{3}(x, y)=D_{(\gamma)} A_{\mu}(x)+\sum_{n=0}^{\infty} D_{(n)} \sin \left(m_{Z_{n}^{\prime}} y+\phi_{n}\right) Z_{n, \mu}^{\prime}(x),
$$

where $D_{(\gamma, n)}$ is a normalization factor, the masses $m_{Z_{n}^{\prime}}$ of the $Z^{\prime}$ states are the solutions of

$$
\left(m_{Z_{n}^{\prime}}^{2}-\frac{g^{2} g^{\prime 2}}{g_{5}^{4}}\right) \tan \left(m_{Z_{n}^{\prime}} \pi R\right)=\frac{\left(g^{2}+g^{\prime 2}\right)}{g_{5}^{2}} m_{Z_{n}^{\prime}},
$$

and the phase constant $\phi_{n}$ satisfies

$$
m_{Z_{n}^{\prime}} \tan \phi_{n}=-\frac{g^{\prime 2}}{g_{5}^{2}} .
$$

In the Kaluza-Klein expansion (4.6) we have separated out the $m_{Z_{n}^{\prime}}=0, \phi_{n}=\pi / 2$ solution of equations (4.7) and (4.8) as the massless photon $A$. The next lightest state is just the standard model $Z \equiv Z_{0}^{\prime}$, with mass $m_{Z} \approx\left(g^{2}+g^{\prime 2}\right)^{1 / 2} /\left(g_{5} \sqrt{\pi R}\right)$. The remaining states have masses of approximately

$$
m_{W_{n}^{\prime}}=m_{Z_{n}^{\prime}}=\frac{n}{R},
$$

up to corrections of $\mathcal{O}\left(g^{2}\left(\pi R / g_{5}^{2}\right)\right)$.

The equations (4.5) and (4.7) which determine the masses of the Kaluza-Klein towers are the same as those derived in Ref. [1], in a five-dimensional $S_{1} / Z_{2}$ orbifold theory with non-trivial kinetic terms at the orbifold fixed points. ${ }^{\ddagger}$ It is the presence of these non-trivial kinetic terms at the boundaries which shift the masses of the Kaluza-Klein vector bosons so that the lightest states can be identified as the standard model $W$ and $Z$ bosons (and photon). We note that these non-trivial boundary terms interpolate between the standard Neumann $\left(\partial_{5} W=0\right)$ and Dirichlet $(W=0)$ conditions at the boundaries. For instance, if $g \rightarrow 0$, the solutions to (4.5) correspond to Dirichlet boundary conditions at $y=\pi R$ (with the $W^{ \pm} \equiv W_{0}^{\prime \pm}$ mode becoming massless and decoupling), whereas if $g \rightarrow \infty$, they correspond to Neumann boundary conditions at $y=\pi R$. In particular, if we let $g \rightarrow \infty$ and $q^{\prime} \rightarrow \infty$ we recover the toy $S U(2) \rightarrow U(1)$ model of Ref. [6].

${ }^{\ddagger}$ Our equations actually differ from Ref. 11 by a factor of 2 in the boundary couplings, which just corresponds to a different treatment of the $\delta$-function at the boundary. The $\delta$-function in Ref. 11 can be considered as a limiting case of a finite-size brane which straddles the orbifold fixed point, whereas the $\delta$-function in eq. (4.3) lies entirely within the interval. 


\section{Coupling to matter fields and constraints from experiment}

In the $U(1) \times[S U(2)]^{N} \times S U(2)_{N+1}$ model the couplings $g$ and $g^{\prime}$ are approximately equal to the weak $S U(2)_{L}$ and hypercharge $U(1)_{Y}$ couplings in the standard model, respectively. Therefore, the most obvious choice for incorporating matter fermions in this model is to couple the left-handed fermions to the $S U(2)_{N+1}$ and the left- and right-handed fermions to the $U(1)$ with charges $Y_{L}$ and $Y_{R}$, respectively, as in the standard model. Mass terms for the fermions can be written in the form

$$
\mathcal{L}_{\text {mass }}=-h f \bar{\psi}_{R} \Sigma_{1} \Sigma_{2} \cdots \Sigma_{N+1} \psi_{L}+\text { h.c. },
$$

where $h$ is the appropriate Yukawa coupling, and $\bar{\psi}_{R}=\left(\bar{u}_{R}, 0\right)$ for up-type fermions and $\bar{\psi}_{R}=\left(0, \bar{d}_{R}\right)$ for down-type fermions. Even though the $\bar{u}_{R}$ and $\bar{d}_{R}$ fields are not connected by an $S U(2)$ symmetry, it is still useful to maintain the 2-component notation for the righthanded fields, since the $U(1)$ that is coupled to $\Sigma_{1}$ is treated as a subgroup of a global $S U(2)$. Under the $U(1)$ symmetry $\bar{\psi}_{R}$ has a charge $-Y_{R}=-Q_{E M}=-\left(T_{3}+Y_{L}\right), \Sigma_{1}$ has a charge of $T_{3}$, and $\psi_{L}$ has a charge of $Y_{L}$; thus, the mass term is invariant under the $U(1)$ symmetry, in addition to the chain of $S U(2)$ symmetries. The extra-dimensional interpretation of the $N+1 \rightarrow \infty$ limit is not particularly simple for this manner of incorporation of fermions, since the left-handed fermions would necessarily have non-zero wave functions on both the $y=0$ and $y=\pi R$ branes, but would have zero wave functions in the bulk. In addition, the mass term (5.1) would involve a nonlocal gauge Wilson loop extending between the two branes. Nevertheless, the $N+1 \rightarrow \infty$ limit is well-defined.

Incorporating the fermions into the model in this manner, we can now investigate what are the experimental constraints on it. We first consider the direct constraints from producing the heavy gauge bosons at colliders and then consider the indirect constraints from precision electroweak measurements.

The coupling of fermions to gauge bosons can be written

$$
\mathcal{L}_{\text {Int }}=g^{\prime} \bar{\psi} \gamma_{\mu}\left(Y_{L} P_{L}+Y_{R} P_{R}\right) \psi B^{\mu}+g \bar{\psi} \gamma_{\mu} T^{a} P_{L} \psi W_{N+1}^{a \mu},
$$

where, $P_{R, L}$ are the projection operators, $\left(1 \pm \gamma_{5}\right) / 2$. By expressing $B^{\mu}$ and $W_{N+1}^{a \mu}$ in terms of the mass eigenstates using equations (2.4) and (2.5), we find that compared to the $W$ and $Z$ couplings to fermions, the $W_{k}^{\prime}$ and $Z_{k}^{\prime}$ couplings are down by factors of $\mathcal{O}(\lambda) \approx \mathcal{O}\left(m_{W} / m_{W_{1}^{\prime}}\right)$. Due to this suppression, the direct production mass bounds at a collider are significantly weakened. The most significant bounds on the $W_{1}^{\prime}$ and $Z_{1}^{\prime}$ masses come from the Tevatron and LEP II, respectively. The derivation of a detailed bound is beyond the scope of this work and we content ourselves with obtaining the following estimates.

The Tevatron (CDF) limit on a $W^{\prime}$ that couples with Standard Model strength is presented in Fig. 2 of Ref. [12]. In our case, the ratio $\sigma\left(q \bar{q} \rightarrow W_{1}^{\prime} \rightarrow \ell \nu\right) / \sigma(q \bar{q} \rightarrow W \rightarrow \ell \nu)$ is suppressed by an additional factor of $\left|a_{(N+1) 1}\right|^{2} \approx k\left(m_{W}^{2} / m_{W_{1}^{\prime}}^{2}\right)$ to that shown in the plot, where $k=1$ for $N=1$ and $k=2$ for $N \rightarrow \infty$. By rescaling the cross sections shown in that figure, we estimate that the corresponding limits in our case would be about 
$m_{W_{1}^{\prime}} \gtrsim 500 \mathrm{GeV}$ for $N \rightarrow \infty$, with the limit for $N=1$ weaker by about $50 \mathrm{GeV}$. We should note that in our models, the $W_{1}^{\prime}$ also decays to $W Z$ with roughly the same coupling strength as it does to fermions; this may alter the analysis further.

The LEP II bound on new four fermion contact interactions are presented (for the case of strong coupling) in Ref. [13] by making fits to $\sigma\left(e^{+} e^{-} \rightarrow f \bar{f}\right)$. This can be translated to a bound on $m_{Z_{1}^{\prime}}$ since a heavy $Z^{\prime}$ effectively induces a four fermion contact interaction. Extracting the relevant contact interactions induced in our model, and comparing to the results of the LEP II analysis, we estimate that the mass bound is about $m_{Z_{1}^{\prime}} \gtrsim 480 \mathrm{GeV}$ for $N \rightarrow \infty$ and $m_{Z_{1}^{\prime}} \gtrsim 400 \mathrm{GeV}$ for $N=1$.

The indirect influence of new physics on electroweak processes can be fully parametrized in terms of the oblique parameters $S, T$, and $U$ [14], as long as the light fermions do not couple directly to the new, heavy gauge bosons. In our model such coupling does occur, but it is suppressed by a factor $\lambda$ in each vertex, in addition to the $\lambda^{2}$ suppression that comes from the large mass in the gauge boson propagators at low energies. Therefore, the dominant electroweak corrections in our model, which are $\mathcal{O}\left(\lambda^{2}\right)$, can be obtained purely from the couplings of fermions to the standard model $W$ and $Z$ bosons, both at low energies and at the $Z$-pole, without considering the direct fermion coupling to the heavier mass eigenstates. Deviations from standard model relations in the $W$ and $Z$ couplings can then be parametrized in terms of $S, T$, and $U$. Following Ref. [15], we have expressed the charged-current and neutral-current interactions for the physical $W$ and $Z$ bosons, obtained from eq. (5.2), in terms of the electric charge $e$, the $Z$ boson mass $m_{Z}$, and the Fermi constant $G_{F}$, plus new physics contributions. Comparing to the general expressions in Ref. [15], we obtain

$$
\begin{aligned}
\alpha S & =\frac{2 N(N+2)}{3(N+1)} \frac{\lambda^{2} \lambda^{\prime 2}}{\lambda^{2}+\lambda^{\prime 2}} \\
& =\frac{8 N(N+2)}{3}\left(\sin \frac{\pi}{2(N+1)}\right)^{2} \frac{m_{W}^{2}}{m_{W_{1}^{\prime}}^{2}} \sin ^{2} \theta_{W}, \\
T & =0 \\
U & =0 .
\end{aligned}
$$

The vanishing of $T$ and $U$, at this order, is a consequence of the coupling of $B_{\mu}$ as the $T^{3}$ component of a global $S U(2)$, a choice which preserves an approximate custodial symmetry. For the minimal $(N=1)$ model we have

$$
\alpha S=4 \frac{m_{W}^{2}}{m_{W_{1}^{\prime}}^{2}} \sin ^{2} \theta_{W},
$$

whereas a true fifth dimension $(N \rightarrow \infty)$ gives

$$
\alpha S=\frac{2 \pi^{2}}{3} \frac{m_{W}^{2}}{m_{W_{1}^{\prime}}^{2}} \sin ^{2} \theta_{W} .
$$

It is also possible to obtain these corrections by directly applying the definition of $S, T$, and $U$ in terms of gauge-eigenstate vacuum polarizations, as given in Ref. [14]. Since this 

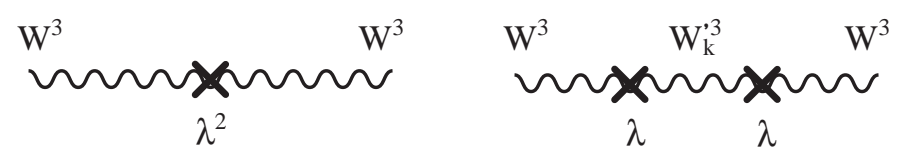

Figure 8: Contributions to $\Pi_{33}\left(q^{2}\right)$ from mixing of the gauge bosons at order $\lambda^{2}$.

offers some insight into the origin of the corrections, we give an overview of this approach here. At tree level the fermions couple only to the gauge eigenstates $W_{N+1}^{a}$ and $B$, so all interactions can be calculated using the full propagators of these gauge eigenstates. In this sense the re-diagonalization of the gauge eigenstates into mass eigenstates can be considered purely "oblique"; i.e., it only modifies the gauge-eigenstate propagators and does not give any "direct" vertex corrections. We can calculate these tree-level oblique corrections as a perturbation expansion in $\lambda$ and $\lambda^{\prime}$, in exact analogy to the loop-level perturbative corrections that occur in the standard model. The parameter $S$ is defined by

$$
\alpha S=4 e^{2}\left[\Pi_{33}^{\prime}(0)-\Pi_{3 Q}^{\prime}(0)\right],
$$

where $\Pi_{X Y}^{\prime}\left(q^{2}\right)$ is related to the vacuum-polarization amplitude between the gauge-eigenstate currents $J_{X}$ and $J_{Y}$ by $\Pi_{X Y}\left(q^{2}\right)=\Pi_{X Y}(0)+q^{2} \Pi_{X Y}^{\prime}\left(q^{2}\right)$. At zeroeth order in $\lambda$ and $\lambda^{\prime}$, the standard model $W^{ \pm}, Z$, and photon are obtained from linear combinations of the "brane" gauge bosons $W_{N+1}^{a}$ and $B$. Meanwhile, the remaining "bulk" gauge eigenstates $W_{i}^{a}$ are diagonalized into mass eigenstates $W_{j}^{\prime a}$ by $W_{i}^{a}=\sum a_{i j} W_{j}^{\prime a}$ for $i, j=1$ to $N$. The masses of these states, $m_{W_{j}^{\prime}}$, and the mixings, $a_{i j}$, can be found from the $\lambda=\lambda^{\prime}=0$ limit of the masses and mixings of the heavy gauge boson states, given in appendix B. Contributions to the $\Pi$ 's then arise at $\mathcal{O}\left(\lambda^{2}\right)$ via the perturbative mixing between the "bulk" and "brane" gauge bosons. For example, the Feynman diagrams for the contribution to $\Pi_{33}$ are shown in Fig. 8, where the mass insertions come from the terms in the mass matrix of eq. (B.8) which are proportional to $\lambda^{2}$ and $\lambda$. Noting that the mass insertion proportional to $\lambda$ only has couplings between the $W_{N+1}^{3}$ and $W_{N}^{3}$ gauge eigenstates, we obtain

$$
i g^{2} \Pi_{33}\left(q^{2}\right)=\left\{\frac{i \lambda^{2} \tilde{g}^{2} f^{2}}{4}+\sum_{k=1}^{N}\left(\frac{-i \lambda \tilde{g}^{2} f^{2}}{4}\right)\left(\frac{-i\left|a_{N k}\right|^{2}}{q^{2}-m_{W_{k}^{\prime}}^{2}}\right)\left(\frac{-i \lambda \tilde{g}^{2} f^{2}}{4}\right)\right\} .
$$

where we have expanded the $\left\langle W_{N}^{3} W_{N}^{3}\right\rangle$ propagator in terms of the mass eigenstate propagators. In calculating $\Pi_{3 Q}$ we also need $\left\langle W_{N}^{3} W_{1}^{3}\right\rangle$, whose expansion in terms of the mass eigenstate propagators involves the coefficients $a_{N k} a_{1 k}^{*}$. This leads to the following expression:

$$
\Pi_{33}^{\prime}(0)-\Pi_{3 Q}^{\prime}(0)=\frac{\tilde{g}^{2} f^{4}}{16}\left[-\sum_{k=1}^{N} \frac{\left|a_{N k}\right|^{2}}{m_{W_{k}^{\prime}}^{4}}+\sum_{k=1}^{N} \frac{a_{N k}\left(a_{1 k}^{*}+a_{N k}^{*}\right)}{m_{W_{k}^{\prime}}^{4}}\right] .
$$

Thus, we find a nonzero contribution to $S$ from the presence of the $a_{N k} a_{1 k}^{*}$ terms, which do not cancel between $\Pi_{33}^{\prime}(0)$ and $\Pi_{3 Q}^{\prime}(0)$. Using the formulae in Appendix B and some algebraic identities, we then recover the results of Eq.(5.3)..$^{\S}$

\footnotetext{
${ }^{\S}$ In this approach we note that any other oblique parameters which might distinguish between low-energy and $Z$-pole processes, such as those considered in Ref. [16], have an additional $\lambda^{2}$ suppression factor, as expected from preceding arguments.
} 
Recent experimental constraints on $S$ and $T$ have been compiled in Ref. [17], where the limits are given as a function of the Higgs boson mass. In principle, its contributions must be subtracted from the above $S$ and $T$ parameters, since there is no Higgs boson in our model. However, given that the dependence on $m_{H}$ is not too large, we can still obtain an estimate of how these constraints impact our model. For $m_{H}=600 \mathrm{GeV}$ with the constraint $S \geq 0$, and using Bayesian statistics, the limit on $S$ is $S \leq 0.14$. This result corresponds to $m_{W_{1}^{\prime}} \gtrsim 2.3 \mathrm{TeV}(N=1)$ and $m_{W_{1}^{\prime}} \gtrsim 3 \mathrm{TeV}(N \rightarrow \infty)$. Unfortunately, for models in which the first $W^{\prime}$ mass is so large, unitarity will be violated even before the scale of the first $W^{\prime}$ is reached. Therefore, it appears that the method used in this section to incorporate matter fermions into the model is not viable.

\section{Conclusions}

In this paper we have investigated a class of theory-space models, which consist of a chain of $S U(2)$ gauge theories and one $U(1)$ gauge theory linked together by non-linear sigma fields, and which contain no physical Higgs scalar. By allowing the couplings of the gauge fields at the first and last site to differ from the others, we can obtain the $W^{ \pm}$and $Z$ boson masses for the first charged and neutral states in a tower of massive vector bosons (in addition to a massless state, the photon). Furthermore, we have found that the unitarity violation in $W_{L}^{+} W_{L}^{-}$scattering, which one finds in the standard model without a physical Higgs boson, is delayed by the exchange of the tower of heavy vector boson states [5].

In the $N+1 \rightarrow \infty$ limit of these models, we find that the coefficient of the leading $E^{2} / m_{W}^{2}$ term in the scattering amplitude vanishes. In this limit, these models become an $S U(2)$ gauge theory defined on a fifth-dimensional line segment, with the $S U(2)$ broken explicitly to $U(1)$ at one boundary, and with nontrivial kinetic terms at both boundaries. The effective four-dimensional gauge sector reduces to the standard model gauge sector at low energies and can be made unitary to scales of $10 \mathrm{TeV}$ or more. At the gauge-sector level, this model is simpler than those proposed previously [6, 0, 8], all of which were based on the gauge group $S U(2) \times S U(2) \times U(1)$.

We also considered a particular implementation of matter fermions coupled to these models. In this implementation the fermions only couple directly to the first and last gauge fields in the chain. This naturally gives standard-model-like couplings of the fermions to the $W^{ \pm}$and $Z$ bosons, while the couplings to the heavier vector bosons are suppressed by ratios of the $W$ mass to the mass of the next higher $W^{\prime}$ state. In this way the bounds on the $W^{\prime}$ and $Z^{\prime}$ masses are lowered compared to models where the $W^{\prime}$ and $Z^{\prime}$ have standard-model-like couplings.

We found that the most serious constraint on this particular implementation of fermions comes from electroweak precision measurements. Due to the mixing between the vector bosons, there is a small deviation from the standard model prediction, which can be parameterized (to first order in $m_{W}^{2} / m_{W_{1}^{\prime}}^{2}$ ) in terms of the oblique correction parameters $S$, $T$, and $U$. In our models we find $T=U=0$ occurs naturally, because of an approximate custodial $S U(2)$. This occurs without the need to introduce an additional $S U(2)$ gauge field in the bulk as in refs. [6, 7, 8]; in our models the already-present $S U(2)$ gauge 
symmetry at adjacent positions in the latticized fifth dimension provides the necessary custodial symmetry. However, there is a reasonably-sized contribution to $S$. In fact, the electroweak precision constraints on $S$ probably force the scale of $m_{W_{1}^{\prime}}$ to be too large to fix the unitarity problem which initially motivated our investigation.

This non-zero contribution to $S$ has also been found in other attempts at Higgsless models of electroweak-symmetry breaking [8]. It is interesting that these models, which appear similar to technicolor models in many ways, should have the same difficulty with the $S$ parameter that plagued the simplest versions of technicolor [14]. However, these models offer a new approach to this problem, with the possibility to find a mechanism to make $S$ "naturally" small.

Finally, we note that the implementation of the matter fields presented in this paper does not have a simple extra-dimensional interpretation in the $N+1 \rightarrow \infty$ limit. The difficulty arises because the left-handed fields have non-zero hypercharge $\left(Y_{L}\right)$, and therefore must couple at both ends of the fifth-dimensional interval. Even if we let the fermions extend into the bulk (thereby allowing a local mass term), we find that the fermions at all positions along the line segment must couple to $W^{3}(x, y=0)$, due to the non-zero $Y_{L}$. Although this may be considered purely a question of aesthetics, since the present models are no less well-defined than a five-dimensional theory, it is still worthwhile to consider this issue and think of other generalizations.

\section{Acknowledgments}

This work was supported by the US National Science Foundation under grants PHY0070443 and PHY-0244789. We would like to thank Sekhar Chivukula and Tim Tait for useful discussions.

\section{A. Solutions for the $U(1) \times S U(2)_{1} \times S U(2)_{2}$ Model}

In this model there are five independent parameters: $g^{\prime}, \tilde{g}, g, f_{1}, f_{2}$. We can rewrite these as functions of the standard model parameters $e, m_{W}, m_{Z}$ and the masses of the heavy vector bosons, $m_{W^{\prime}}, m_{Z^{\prime}}$. We obtain

$$
\begin{aligned}
g^{\prime 2} & =\frac{e^{2} m_{Z}^{2} m_{Z^{\prime}}^{2}}{m_{W}^{2} m_{W^{\prime}}^{2}} \\
\tilde{g}^{2} & =g^{\prime 2}\left[\frac{\left(m_{W}^{2}+m_{W^{\prime}}^{2}\right)\left(m_{Z}^{2}+m_{Z^{\prime}}^{2}-m_{W}^{2}-m_{W^{\prime}}^{2}\right)+m_{W}^{2} m_{W^{\prime}}^{2}-m_{Z}^{2} m_{Z^{\prime}}^{2}}{\left(m_{Z}^{2}+m_{Z^{\prime}}^{2}-m_{W}^{2}-m_{W^{\prime}}^{2}\right)^{2}}\right] \\
g^{2} & =g^{\prime 2} m_{W^{\prime}}^{2} m_{W^{\prime}}^{2}\left[\frac{\left(m_{W}^{2}+m_{W^{\prime}}^{2}\right)\left(m_{Z}^{2}+m_{Z^{\prime}}^{2}-m_{W}^{2}-m_{W^{\prime}}^{2}\right)+m_{W^{\prime}}^{2} m_{W^{\prime}}^{2}-m_{Z^{\prime}}^{2} m_{Z^{\prime}}^{2}}{\left(m_{Z}^{2}-m_{W}^{2}\right)\left(m_{Z^{\prime}}^{2}-m_{W^{\prime}}^{2}\right)\left(m_{Z^{\prime}}^{2}-m_{W}^{2}\right)\left(m_{W^{\prime}}^{2}-m_{Z}^{2}\right)}\right] \\
f_{1}^{2} & =\frac{4}{g^{\prime 2}}\left(m_{Z}^{2}+m_{Z^{\prime}}^{2}-m_{W}^{2}-m_{W^{\prime}}^{2}\right) \\
f_{2}^{2} & =\frac{16 m_{W}^{2} m_{W^{\prime}}^{2}}{\tilde{g}^{2} g^{2} f_{1}^{2}} .
\end{aligned}
$$

Note these equations imply the relationship $m_{Z}^{\prime}>m_{W}^{\prime}$. 
The charged boson mixing matrix, defined in Eq. (2.4), is given by $a_{11}=a_{22}=\cos \phi$ and $a_{12}=-a_{21}=\sin \phi$ with

$$
\begin{aligned}
\cos \phi & =\left[\frac{m_{W^{\prime}}^{2}\left(m_{W^{\prime}}^{2}-m_{Z}^{2}\right)\left(m_{Z^{\prime}}^{2}-m_{W^{\prime}}^{2}\right)}{m_{W^{\prime}}^{2}\left(m_{W^{\prime}}^{2}-m_{Z}^{2}\right)\left(m_{Z^{\prime}}^{2}-m_{W^{\prime}}^{2}\right)+m_{W}^{2}\left(m_{Z^{\prime}}^{2}-m_{W}^{2}\right)\left(m_{Z}^{2}-m_{W}^{2}\right)}\right]^{1 / 2}, \\
\sin \phi & =\left[\frac{m_{W}^{2}\left(m_{Z^{\prime}}^{2}-m_{W}^{2}\right)\left(m_{Z}^{2}-m_{W}^{2}\right)}{m_{W^{\prime}}^{2}\left(m_{W^{\prime}}^{2}-m_{Z}^{2}\right)\left(m_{Z^{\prime}}^{2}-m_{W^{\prime}}^{2}\right)+m_{W}^{2}\left(m_{Z^{\prime}}^{2}-m_{W}^{2}\right)\left(m_{Z}^{2}-m_{W}^{2}\right)}\right]^{1 / 2} .
\end{aligned}
$$

The neutral boson mixing matrix, defined in Eq. (2.5), is given by

$$
b_{00}=e / g^{\prime}, \quad b_{10}=e / \tilde{g}, \quad b_{20}=e / g
$$

and

$$
\begin{aligned}
b_{01}= & -\left[\frac{\left(m_{Z^{\prime}}^{2}-m_{W}^{2}\right)\left(m_{Z^{\prime}}^{2}-m_{W^{\prime}}^{2}\right)}{m_{Z^{\prime}}^{2}\left(m_{Z^{\prime}}^{2}-m_{Z}^{2}\right)}\right]^{1 / 2}, \\
b_{11}= & {\left[\frac{\left(m_{Z^{\prime}}^{2}-m_{W}^{2}\right)\left(m_{Z^{\prime}}^{2}-m_{W^{\prime}}^{2}\right)}{m_{Z^{\prime}}^{2}\left(m_{Z^{\prime}}^{2}-m_{Z}^{2}\right)\left[\left(m_{W}^{2}+m_{W^{\prime}}^{2}\right)\left(m_{Z}^{2}+m_{Z^{\prime}}^{2}-m_{W}^{2}-m_{W^{\prime}}^{2}\right)+m_{W^{\prime}}^{2} m_{W^{\prime}}^{2}-m_{Z^{\prime}}^{2} m_{Z^{\prime}}^{2}\right]}\right]^{1 / 2} } \\
& \times\left(m_{W^{\prime}}^{2}+m_{W}^{2}-m_{Z}^{2}\right), \\
b_{21}= & -\left[\frac{m_{W^{\prime}}^{2} m_{W^{\prime}}^{2}\left(m_{Z}^{2}-m_{W}^{2}\right)\left(m_{W^{\prime}}^{2}-m_{Z}^{2}\right)}{m_{Z^{\prime}}^{2}\left(m_{Z^{\prime}}^{2}-m_{Z}^{2}\right)\left[\left(m_{W}^{2}+m_{W^{\prime}}^{2}\right)\left(m_{Z}^{2}+m_{Z^{\prime}}^{2}-m_{W}^{2}-m_{W^{\prime}}^{2}\right)+m_{W^{\prime}}^{2} m_{W^{\prime}}^{2}-m_{Z^{\prime}}^{2} m_{Z^{\prime}}^{2}\right]}\right]^{1 / 2}, \\
b_{02}= & -\left[\frac{\left.\left(m_{Z}^{2}-m_{W}^{2}\right)\left(m_{W^{\prime}}^{2}-m_{Z}^{2}\right)\right]^{1 / 2},}{m_{Z}^{2}\left(m_{Z^{\prime}}^{2}-m_{Z}^{2}\right)}\right]^{1 / 2}, \\
b_{12}= & {\left[\frac{\mathrm{A} .4)}{m_{Z}^{2}\left(m_{Z^{\prime}}^{2}-m_{Z}^{2}\right)\left[\left(m_{W}^{2}+m_{W^{\prime}}^{2}\right)\left(m_{Z}^{2}+m_{W}^{2}\right)\left(m_{W^{\prime}}^{2}-m_{W}^{2}-m_{W^{\prime}}^{2}\right)+m_{W}^{2} m_{W^{\prime}}^{2}-m_{Z^{\prime}}^{2} m_{Z^{\prime}}^{2}\right]}\right]^{1 / 2} } \\
& \times\left(m_{W^{\prime}}^{2}+m_{W}^{2}-m_{Z^{\prime}}^{2}\right), \\
b_{22}= & {\left[\frac{m_{W^{\prime}}^{2} m_{W^{\prime}}^{2}\left(m_{Z^{\prime}}^{2}-m_{W}^{2}\right)\left(m_{Z^{\prime}}^{2}-m_{W^{\prime}}^{2}\right)}{m_{Z}^{2}\left(m_{Z^{\prime}}^{2}-m_{Z}^{2}\right)\left[\left(m_{W}^{2}+m_{W^{\prime}}^{2}\right)\left(m_{Z}^{2}+m_{Z^{\prime}}^{2}-m_{W}^{2}-m_{W^{\prime}}^{2}\right)+m_{W}^{2} m_{W^{\prime}}^{2}-m_{Z^{\prime}}^{2} m_{Z^{\prime}}^{2}\right]}\right]^{1 / 2} . }
\end{aligned}
$$




\section{B. Solutions for the $U(1) \times[S U(2)]^{N} \times S U(2)_{N+1}$ Model}

The mass matrix for the charged bosons is

$$
M_{W}^{2}=\frac{\tilde{g}^{2} f^{2}}{4}\left[\begin{array}{ccccccc}
2 & -1 & 0 & \cdots & & & \\
-1 & 2 & -1 & \cdots & & & \\
0 & -1 & 2 & \cdots & & & \\
\vdots & \vdots & \vdots & \ddots & \vdots & \vdots & \vdots \\
& & & \cdots & 2 & -1 & 0 \\
& & & \cdots & -1 & 2 & -\lambda \\
& & & \cdots & 0 & -\lambda & \lambda^{2}
\end{array}\right],
$$

where $\lambda=g / \tilde{g}$. Note that the sequence of $(-12-1)$ in each row of this matrix (except the first and the last two) acts as a discrete second derivative, whose eigenfunction is a sine function. Thus, we obtain as eigenvectors for this matrix

$$
\psi_{(n)}=C_{(n)}\left[\begin{array}{c}
\sin \omega_{(n)} \\
\sin 2 \omega_{(n)} \\
\sin 3 \omega_{(n)} \\
\vdots \\
\sin (N-1) \omega_{(n)} \\
\sin N \omega_{(n)} \\
\frac{1}{\lambda} \sin (N+1) \omega_{(n)}
\end{array}\right]
$$

where $C_{(n)}$ is a normalization constant and the eigenvalues are

$$
m_{W_{n}^{\prime}}^{2}=\tilde{g}^{2} f^{2} \sin ^{2} \frac{\omega_{(n)}}{2} .
$$

The last row of the eigenvector equation $M_{W}^{2} \psi_{(n)}=m_{W_{n}^{\prime}}^{2} \psi_{(n)}$ gives the characteristic equation for this system

$$
\sin ^{2} \frac{\omega}{2} \sin (N+1) \omega=\frac{\lambda^{2}}{4}[\sin (N+1) \omega-\sin N \omega]
$$

which has $N+1$ solutions, $\omega_{(n)}$. Using this equation and trigonometric identities [18], we obtain a simple formula for the normalization constant

$$
C_{(n)}=\left[\frac{N+1}{2}+\frac{\sin \left[2(N+1) \omega_{(n)}\right]}{4 \sin \omega_{(n)}}\right]^{-1 / 2}
$$

Solving Eq. (B.4) perturbatively, and identifying the standard model $W \equiv W_{N+1}^{\prime}$, we obtain for the charged boson masses

$$
\begin{aligned}
m_{W}^{2} & =\frac{g^{2} f^{2}}{4(N+1)}\left(1-\lambda^{2} \frac{N(2 N+1)}{6(N+1)}+\mathcal{O}\left(\lambda^{4}\right)\right) \\
m_{W_{n}^{\prime}}^{2} & =\tilde{g}^{2} f^{2}\left(\sin \frac{n \pi}{2(N+1)}\right)^{2}+2 m_{W}^{2}\left(\cos \frac{n \pi}{2(N+1)}\right)^{2}\left(1+\mathcal{O}\left(\lambda^{2}\right)\right) .
\end{aligned}
$$


The elements of the charged boson mixing matrix are

$$
\begin{aligned}
a_{(N+1)(N+1)} & =1-\lambda^{2} \frac{N(2 N+1)}{12(N+1)}+\mathcal{O}\left(\lambda^{4}\right), \\
a_{n(N+1)} & =\lambda \frac{n}{N+1}+\mathcal{O}\left(\lambda^{3}\right), \\
a_{(N+1) m} & =-\lambda \sqrt{\frac{2}{N+1}} \frac{\sin \frac{\pi m N}{N+1}}{4 \sin ^{2} \frac{\pi m}{2(N+1)}}+\mathcal{O}\left(\lambda^{3}\right), \\
a_{n m} & =\sqrt{\frac{2}{N+1}} \sin \frac{\pi n m}{N+1}+\mathcal{O}\left(\lambda^{2}\right),
\end{aligned}
$$

where $n$ and $m$ run from 1 to $N$.

The mass matrix for the neutral bosons is

$$
M_{Z}^{2}=\frac{\tilde{g}^{2} f^{2}}{4}\left[\begin{array}{ccccccc}
\lambda^{\prime 2} & -\lambda^{\prime} & 0 & \cdots & & & \\
-\lambda^{\prime} & 2 & -1 & \cdots & & & \\
0 & -1 & 2 & \cdots & & & \\
\vdots & \vdots & \vdots & \ddots & \vdots & \vdots & \vdots \\
& & & \cdots & 2 & -1 & 0 \\
& & & \cdots & -1 & 2 & -\lambda \\
& & & \cdots & 0 & -\lambda & \lambda^{2}
\end{array}\right]
$$

where $\lambda^{\prime}=g^{\prime} / \tilde{g}$. The eigenvector equation $M_{Z}^{2} \chi_{(n)}=m_{Z_{n}^{\prime}}^{2} \chi_{(n)}$ can be solved in a similar manner to the charged mass matrix. The eigenvectors are

$$
\chi_{(n)}=D_{(n)}\left[\begin{array}{c}
\frac{1}{\lambda^{\prime}} \sin \phi_{(n)} \\
\sin \left[\rho_{(n)}+\phi_{(n)}\right] \\
\sin \left[2 \rho_{(n)}+\phi_{(n)}\right] \\
\vdots \\
\sin \left[(N-1) \rho_{(n)}+\phi_{(n)}\right] \\
\sin \left[N \rho_{(n)}+\phi_{(n)}\right] \\
\frac{1}{\lambda} \sin \left[(N+1) \rho_{(n)}+\phi_{(n)}\right]
\end{array}\right]
$$

where $D_{(n)}$ is a normalization constant and the eigenvalues are

$$
m_{Z_{n}^{\prime}}^{2}=\tilde{g}^{2} f^{2} \sin ^{2} \frac{\rho_{(n)}}{2} .
$$

The characteristic equation for this system is

$$
\sin ^{2} \frac{\rho}{2} \sin (N+1) \rho=\frac{\lambda^{2}+\lambda^{\prime 2}}{4}[\sin (N+1) \rho-\sin N \rho]+\frac{\lambda^{2} \lambda^{\prime 2}}{4} \sin N \rho
$$

which has $N+2$ solutions, $\rho_{(n)}$. The phase constant $\phi_{(n)}$ satisfies

$$
\tan \phi_{(n)} \tan \frac{\rho_{(n)}}{2}=\frac{\lambda^{\prime 2}}{\lambda^{\prime 2}-2} .
$$


Using Eqs. (B.11) and (B.12), we obtain for the normalization constant

$$
D_{(n)}=\left[\frac{N+1}{2}+\frac{\sin \left[(N+1) \rho_{(n)}\right] \cos \left[(N+1) \rho_{(n)}+2 \phi_{(n)}\right]}{2 \sin \rho_{(n)}}\right]^{-1 / 2}
$$

There is one trivial solution to Eqs. (B.11) and (B.12), which corresponds to the photon solution. Identifying the photon by $A \equiv Z_{0}^{\prime}$, we obtain the solution $\rho_{(0)}=0, \phi_{(0)}=\pi / 2$. The photon is massless, and the electromagnetic coupling is given by

$$
\frac{1}{e^{2}}=\frac{1}{g^{\prime 2}}+\frac{N}{\tilde{g}^{2}}+\frac{1}{g^{2}}
$$

The photon mixing angles are

$$
b_{00}=e / g^{\prime}, \quad b_{n 0}=e / \tilde{g}, \quad b_{(N+1) 0}=e / g
$$

with $n=1$ to $N$.

Identifying the standard model $Z \equiv Z_{N+1}^{\prime}$, we obtain for the the remaining neutral boson masses

$$
\begin{aligned}
m_{Z}^{2} & =\frac{\left(g^{2}+g^{\prime 2}\right) f^{2}}{4(N+1)}\left(1-\left(\lambda^{2}+\lambda^{\prime 2}\right) \frac{N(2 N+1)}{6(N+1)}+\frac{N \lambda^{2} \lambda^{\prime 2}}{\lambda^{2}+\lambda^{\prime 2}}+\mathcal{O}\left(\lambda^{4}\right)\right) \\
m_{Z_{n}^{\prime}}^{2} & =\tilde{g}^{2} f^{2}\left(\sin \frac{n \pi}{2(N+1)}\right)^{2}+2 m_{Z}^{2}\left(\cos \frac{n \pi}{2(N+1)}\right)^{2}\left(1+\mathcal{O}\left(\lambda^{2}\right)\right)
\end{aligned}
$$

The elements of the charged boson mixing matrix are

$$
\begin{aligned}
b_{0(N+1)} & =\frac{-g^{\prime}}{\sqrt{g^{2}+g^{\prime 2}}}\left[1-\left(\lambda^{2}+\lambda^{\prime 2}\right) \frac{N(2 N+1)}{12(N+1)}+\frac{N \lambda^{4}}{2\left(\lambda^{2}+\lambda^{\prime 2}\right)}+\mathcal{O}\left(\lambda^{4}\right)\right] \\
b_{n(N+1)} & =\frac{1}{N+1}\left[n \frac{\lambda^{2}}{\sqrt{\lambda^{2}+\lambda^{\prime 2}}}-(N+1-n) \frac{\lambda^{\prime 2}}{\sqrt{\lambda^{2}+\lambda^{\prime 2}}}+\mathcal{O}\left(\lambda^{3}\right)\right] \\
b_{(N+1)(N+1)} & =\frac{g}{\sqrt{g^{2}+g^{\prime 2}}}\left[1-\left(\lambda^{2}+\lambda^{\prime 2}\right) \frac{N(2 N+1)}{12(N+1)}+\frac{N \lambda^{\prime 4}}{2\left(\lambda^{2}+\lambda^{\prime 2}\right)}+\mathcal{O}\left(\lambda^{4}\right)\right] \\
b_{0 m} & =-\lambda^{\prime} \sqrt{\frac{2}{N+1}} \frac{\sin \frac{\pi m}{N+1}}{4 \sin ^{2} \frac{\pi m}{2(N+1)}}+\mathcal{O}\left(\lambda^{3}\right) \\
b_{n m} & =\sqrt{\frac{2}{N+1} \sin } \frac{\pi n m}{N+1}+\mathcal{O}\left(\lambda^{2}\right) \\
b_{(N+1) m} & =-\lambda \sqrt{\frac{2}{N+1}} \frac{\sin _{\frac{\pi m N}{N+1}}}{4 \sin ^{2} \frac{\pi m}{2(N+1)}}+\mathcal{O}\left(\lambda^{3}\right)
\end{aligned}
$$

where $n$ and $m$ run from 1 to $N$.

Finally, we can use the characteristic equations, (B.4), (B.11), and (B.12), along with the orthonormality of the rows of the $Z^{\prime}$ mixing matrix, to obtain a simple expression for the leading $E^{2} / m_{W_{n}^{\prime}}^{2}$ term in the $W_{n}^{\prime+} W_{n}^{\prime-} \rightarrow W_{n}^{\prime+} W_{n}^{\prime-}$ scattering amplitude, which is the generalization of $R$ in Eq. (3.8). We find

$$
R_{(n)}=C_{(n)}^{4}\left(\frac{m_{W_{n}^{\prime}}^{2}}{f^{2}}\right)\left[\frac{3}{2}(N+1)+\frac{\sin \left[2(N+1) \omega_{(n)}\right]}{\sin \omega_{(n)}}+\frac{\sin \left[4(N+1) \omega_{(n)}\right]}{4 \sin 2 \omega_{(n)}}\right] .
$$


It is interesting to note that this quantity is exactly independent of $g^{\prime}$, and it falls off as $(N+1)^{-2}$ for large $N$. Setting $n=N+1$, we obtain the result for $W^{+} W^{-}$scattering in this model which, to first non-zero order in $\lambda^{2}$, is

$$
R=\frac{g^{2}}{(N+1)^{2}} \text {. }
$$

\section{References}

[1] C. H. Llewellyn Smith, Phys. Lett. B 46, 233 (1973);

D. A. Dicus and V. S. Mathur, Phys. Rev. D 7, 3111 (1973);

J. M. Cornwall, D. N. Levin and G. Tiktopoulos, Phys. Rev. Lett. 30, 1268 (1973)

[Erratum-ibid. 31, 572 (1973)]; Phys. Rev. D 10, 1145 (1974) [Erratum-ibid. D 11, 972

(1975)];

B. W. Lee, C. Quigg and H. B. Thacker, Phys. Rev. Lett. 38, 883 (1977); Phys. Rev. D 16, 1519 (1977);

M. S. Chanowitz and M. K. Gaillard, Nucl. Phys. B 261, 379 (1985).

[2] S. Weinberg, Phys. Rev. D 19, 1277 (1979);

L. Susskind, Phys. Rev. D 20, 2619 (1979); E. Eichten and K. D. Lane, Phys. Lett. B 90, 125 (1980).

[3] Y. Kawamura, Prog. Theor. Phys. 103, 613 (2000) [arXiv:hep-ph/9902423]; Prog. Theor.

Phys. 105, 999 (2001) [arXiv:hep-ph/0012125];

L. J. Hall and Y. Nomura, Phys. Rev. D 64, 055003 (2001) [arXiv:hep-ph/0103125];

A. Hebecker and J. March-Russell, Nucl. Phys. B 613, 3 (2001) [arXiv:hep-ph/0106166];

Nucl. Phys. B 625, 128 (2002) [arXiv:hep-ph/0107039].

[4] C. Csaki, C. Grojean, H. Murayama, L. Pilo and J. Terning, arXiv:hep-ph/0305237.

[5] R. Sekhar Chivukula, D. A. Dicus and H. J. He, Phys. Lett. B 525, 175 (2002)

[arXiv:hep-ph/0111016];

R. S. Chivukula and H. J. He, Phys. Lett. B 532, 121 (2002) [arXiv:hep-ph/0201164].

[6] C. Csaki, C. Grojean, L. Pilo and J. Terning, arXiv:hep-ph/0308038.

[7] Y. Nomura, arXiv:hep-ph/0309189.

[8] R. Barbieri, A. Pomarol and R. Rattazzi, arXiv:hep-ph/0310285.

[9] N. Arkani-Hamed, A. G. Cohen and H. Georgi, Phys. Rev. Lett. 86, 4757 (2001) [arXiv:hep-th/0104005];

C. T. Hill, S. Pokorski and J. Wang, Phys. Rev. D 64, 105005 (2001) [arXiv:hep-th/0104035].

[10] H. Georgi, Nucl. Phys. B 266, 274 (1986).

[11] M. Carena, T. M. P. Tait and C. E. M. Wagner, Acta Phys. Polon. B 33, 2355 (2002) [arXiv:hep-ph/0207056].

[12] T. Affolder et al. [CDF Collaboration], Phys. Rev. Lett. 87, 231803 (2001) [arXiv:hep-ex/0107008].

[13] [ALEPH Collaboration], arXiv:hep-ex/0212036.

[14] M. E. Peskin and T. Takeuchi, Phys. Rev. D 46, 381 (1992). 
[15] C. P. Burgess, S. Godfrey, H. Konig, D. London and I. Maksymyk, Phys. Rev. D 49, 6115 (1994) [arXiv:hep-ph/9312291].

[16] I. Maksymyk, C. P. Burgess and D. London, Phys. Rev. D 50, 529 (1994) [arXiv:hep-ph/9306267].

[17] K. Hagiwara et al. [Particle Data Group Collaboration], Phys. Rev. D 66, 010001 (2002).

[18] I. S. Gradshteyn and I. M. Ryzhik, "Table of Integrals, Series, and Products," $5^{\text {th }}$ edition, Academic Press (1994). 